\title{
Melting is merely skin-thick
}

\section{The phase transition from solid to liquid, perhaps the most puzzling of all, probably begins with surface-melting where the crystallographic orientation is appropriate.}

As phase transitions go, melting is an obscure phenomenon, at least compared with, say, vaporization. Both liquids and solids, being always in equilibrium with their vapours, will, like any condensed phase, disappear into the gas phase given a sufficiently good surrounding vacuum (although that may take a very long time). Liquids, by contrast, appear not to coexist below the melting-point with their solid phases, which serve as nucleation centres for crystal growth. Yet in the absence of nucleation, supercooled liquids are commonplace while superheated solids are not (whence the use by chemists of melting-point measurements as a means of characterizing pure compounds).

These distinctions are not particularly profound in that they are the consequences of spurious circumstances. Condensed phases will evaporate into a good vacuum, for example, because their molecules can get physically away. Moreover, there is ample evidence of what may be called pre-melting as solids are heated towards the melting point; the concentration of interstitial atoms, or of dislocations, can be shown experimentally to increase with increasing temperature inversely with some power of its offset from the melting point, lending support to the notion that melting is just another order-disorder phase transition.

But how is pre-melting to be visualized? As the accumulation of lattice disorders whose effect is to distribute disorder uniformly through the bulk of a crystal? Or as the appearance of possibly evanescent bubbles of liquidity within an ordered crystalline array? One obvious comment on the second possibility is that most liquids are less dense than the solid phases from which they are formed, whence the common supposition (for which the Soviet author H.J. Frenkel was apparently first responsible) that melting is, to begin with, a surface phenomenon.

Here is a neat set of measurements that puts that supposition on a more solid foundation. B. Pluis and three colleagues at the FOM-Institute for Atomic and Molecular Physics at Amsterdam, in the Netherlands, have now described in the most convincing way a set of observations supporting the supposition that the onset of melting is a surface phenomenon - by showing that surface melting is a predictable (or, at least, a post hoc rationalizable) event (Phys. Rev. Lett. 59, 2678; 1987).
What they have done is to show that, at pre-melting temperatures, the disordering of the surface structure is sensitively a function of the crystallographic direction in the underlying lattice of a carefully machined single crystal of metallic lead.

How does one set about revealing such a phenomenon? First find a suitable probe, best visualized as a physical device (say a hat-pin), whose penetration of the supposedly solid object of the study is sensitively a function of its orientation. For practical reasons (as even the most slender hat-pins are too thick for comfort), the authors of this study have used beams of $75.6 \mathrm{keV}$ protons (for practical purposes, billiard balls at that energy) which are known to be virtually unable to penetrate oriented lead crystals when fired at them in the [101] direction, which coincides with the orientation of parallel linear arrays of lead atoms. For a well-ordered lead crystal, the scattering of protons in a roughly perpendicular direction is only small — but is also highly sensitive to the blocking effect of the ordered array of lead atoms.

What the group at Amsterdam has done is to machine a tiny cylindrical sample from a single crystal of lead whose orientation is almost acurately known. They have mounted their piece of lead in such a way that it can be moved transversely to the plane defined by the blocking direction [101] and the nearly-perpendicular direction [121], the former of which is defined by the proton beam and the latter by that of the line to the proton detector.

As the geometry of the experiment is defined, different distances away from the thickest peak of the lead cylinder correspond to particular crystallographic directions, while it is possible to calculate the average penetration depth (in atomic spacings) along the [101] direction at each point. For all places on the surface of the cylindrical sample, the penetration of the proton beam appears to have increased rapidly within 100 degrees of the meltingpoint (roughly $600 \mathrm{~K}$ ), from less than two atomic spacings to nearly 10 . More significantly, the penetration depth seems to be exquisitely sensitive to the crystallographic orientation of different patches on the surface of the lead crystal with respect to the underlying crystallographic axes.

Nobody will be much surprised by this. The parts of a solid crystal at which the number of atoms per unit surface area are the smallest are likely to be those at which disorder will most easily set in, or be observed. The interest in what Pluis et al. have done is in relating their observations of proton scattering to earlier broodings about "surface free energy", as it is called. Here again the analogy between evaporation and melting is appropriate.

It is common, in the discussion of the thermodynamics of the equilibrium with each other of small liquid droplets, to take the view that the free energy of atoms in a droplet and in bulk liquid is the same, but that the formation of droplets may entail additional "surface free-energy" (which is nothing other than the diminished aggregate capacity of a small drop to hold onto peripheral atoms because the van der Waals forces do not saturate). That explains why large drops often grow at the expense of small by transfer through the vapour phase.

If the surface is considered as a whole, this is nothing other than a way of saying that the total free engergy per atom is modified by some small quantity proportional to the cube root of the square of the total bulk. Pedantically, however, none of this allows the legitimate definition of a single free energy of a water molecule in a water droplet, or of a lead atom in a small crystal, which is then modified for the small structure as a whole by a surface-free energy (per unit of area) for some hypothetical surface. Nor does it allow the definition, per atom, of the free energy of a single atom in the surface of a crystal with a particular orientation.

But it is a legitimate subject for inquiry to ask whether the total energy of the microsystem would decrease if the atom were at one place rather than the other. The unconscious error is that of mistaking equilibrium processes, of which free energy is the determinant, for dynamic processes such as those involved in evaporation and, for that matter, melting.

What Pluis et al. have nevertheless shown is that there is indeed a correlation between computed surface free-energy per atom of atoms in surfaces of different orientation, as derived from vapour pressure measurements of lead crystals, and the likelihood that surfaces of the same orientation will show disordered behaviour near the melting point. That also points to the need for a better understanding of the dynamics of pre-melting. Perhaps people will in the end be thrown back onto molecular dynamics.

John Maddox 